\title{
Homogeneous pattern selection and director instabilities of nematic liquid crystal polymers induced by elongational flows
}

\author{
M. Gregory Forest \\ Department of Mathematics, University of North Carolina, Chapel Hill, North Carolina 27599-3250 \\ Qi Wang \\ Department of Mathematical Sciences, Indiana University-Purdue University Indianapolis, Indianapolis, \\ Indiana 46202 \\ Hong Zhou \\ Department of Mathematics, University of California, Santa Cruz, California 95064
}

(Received 23 April 1998; accepted 3 December 1999)

We characterize homogeneous patterns, their stability, and phase transitions in nematic liquid crystal polymers (LCPs) with imposed elongational flows. We combine the flow-induced analysis of order parameters by See et al. [J. Chem. Phys. 92, 792 (1990)], Bhave et al. [J. Rheol. 37, 413 (1993)], Rey [Macromol. Theory Simul. 4, 857 (1995)], and Wang [J. Non-Newtonian Fluid Mech. 22, 147 (1997)], with the pure nematic, full tensor analysis of Shimada et al. [J. Chem. Phys. 88, 7181 (1988)]. To make contact with these seminal studies, we select a moment-averaged Doi kinetic model for flows of rod-like nematic LCPs with a quartic short-range intermolecular potential; the connection with alternative kinetic and continuum models for flows of LCPs is noted. New elongation-induced director instabilities are revealed for patterns previously identified as candidates for stable pattern selection. From a full tensor analysis, we determine the complete phase diagram for homogeneous patterns in the parameter space of LCP concentration and elongation rate. With respect to experimental predictions, in axial extension, biaxial patterns exist but they are all unstable and the only stable patterns are uniaxial; in planar extension, above a moderate concentration the only stable nematic patterns are biaxial. (C) 2000 American Institute of Physics.

[S1070-6631(00)03003-8]

\section{INTRODUCTION}

Nematic liquid crystal polymers (LCPs) in a flow field may exhibit either uniaxial or biaxial symmetry at different locations and time depending on the nature of the flow field and the polymer concentration. Nematic orientation is a mesoscale property, reflective experimentally of averages over a cloud of rod-like molecules. A uniaxial symmetry obtains when there is a single preferred direction of orientation, defining a unique director, with isotropic order in the plane orthogonal to that director; a single order parameter provides the averaged degree of order with respect to the distinguished director. Biaxial symmetry occurs when there is no plane of isotropy, but rather three principal axes of mesoscale orientation, and the degrees of orientation with respect to each principal axis are distinct.

Certain orientation patterns are consistently measured in steady flow processes, presumably consisting in local spatial regions of stable equilibria that respond to the particular flow in that region. In these local regions, the LCP may be reasonably approximated as a spatially homogeneous LCP. There are several competing models for flows of LCPs; the texts by Beris and Edwards ${ }^{1}$ and Larson $^{2}$ provide an excellent summary of both continuum and kinetic theories.

We select for this study the short-range elasticity theory of Doi, ${ }^{3}$ which is a moment-averaged kinetic theory model based on the Doi closure approximation. In this mesoscale theory, the orientation variable is the average with respect to the probability distribution function of the second moment of the molecule axis, yielding an orientation tensor $Q$ which is rank 2, symmetric, and traceless. There is ample evidence that in elongational flows this approximate theory provides a good model for flow-induced patterns and phenomena, including homogeneous phase transitions and fiber flows. ${ }^{4-9}$ The reader is referred to Feng and Leal ${ }^{10-12}$ and Wang ${ }^{13}$ for comparisons of the Doi model employed below and various alternatives in elongational flows.

The upshot of these studies is that the standard Doi closure model with short-range elasticity provides a quite reasonable model for the purposes stated here: homogeneous pattern selection in an imposed elongational flow. In weaker flows, such as imposed shear, the predictions of Doi-type models are far more sensitive to the type of closure approximation. ${ }^{14}$ We note further that the mesoscale averaged model that arises from a Doi-Edwards kinetic theory is qualitatively very similar to continuum models developed by Beris and Edwards, for example. A proper choice of parameters allows one to relate the two models (see pp. 556-559 of Ref. 1). While quantitative predictions made in the following are specific to the Doi closure model, qualitatively similar results are obtained from continuum models and other moment-averaged kinetic models. Alternative studies of flow-induced patterns and stability can be found in the works 
of Khoklov and Semenov, ${ }^{15}$ Wang and Gelbart, ${ }^{16}$ and Hu and Ryskin $^{17,18}$ which employ Onsager-type models.

Physical applications have motivated earlier studies of patterns in imposed elongation. ${ }^{4,10-12,8,9,13}$ In extrusion manufacturing the flow away from boundaries is well approximated locally by unidirectional elongation; in film and sheet manufacturing, or in squeezing flow between parallel disks, there are interior flow regions approximated by planar elongation. Patterns that form are likely to have orientation structure that is locally built from equilibria of LCPs with short-range intermolecular potentials in pure elongational flow.

The present study is restricted to this special flownematic physics as a model for which we can develop a detailed understanding of the nematodynamic response to elongational flow. We provide new information beyond that presented in earlier seminal studies on flow-induced homogeneous patterns. ${ }^{4,6,9,13,16-18}$ In particular we analyze the full orientation tensor, extending the uniaxial results of Refs. 9 and 13 and the biaxial order parameter results of Ref. 8, providing a complete phase diagram: all flow-induced homogeneous equilibria and their stability; all phase transitions; and the orientation modes of every instability.

Our linearized analysis is similar to the full tensor analytical treatment of Shimada et al. ${ }^{19}$ who worked with the kinetic theory for nematic LCPs without flow to elucidate the Doi model description of the isotropic-nematic phase transition. The analysis of Ref. 19 was reproduced in the Doi closure approximation in Ref. 6 to analyze both the istropicnematic and nematic-nematic phase transitions without flow. The key observation in Ref. 19 is an explicit basis of tensorial eigenfunctions of the linearized Doi model, and the key observation here is that with minor modification this same tensorial basis allows us to explicitly solve, in closed form, the elongation-driven linearized stability equations about all homogeneous uniaxial and biaxial equilibria!

The present study applies at a mesoscopic scale set by the moment averaging with respect to the probability distribution function, and where polymer-polymer excluded volume interactions are presumed to dominate pattern selection. At a larger mesoscopic scale, a variety of patterns and textures are observed, where long-range Frank elasticity surely is important, and defects mediate the different local patterns. We refer to the work of Larson and Mead, ${ }^{20}$ Larson and Doi, ${ }^{21}$ Tsuji and Rey, ${ }^{22}$ and Kawaguchi and Denn ${ }^{23}$ for studies aimed at resolving textures, which require a layer of complexity beyond that considered here.

We now set the precise foundation for this paper. Doi $^{24,25}$ and Bhave et al. ${ }^{4}$ developed a kinetic theory for flows of spatially homogeneous LCPs in a Newtonian solvent, subject to an anisotropic hydrodynamic drag and a polymer-polymer mean-field (short-range) interaction with Maier-Saupe potential. See et al. ${ }^{9}$ Bhave et al. ${ }^{4}$ Rey, ${ }^{8}$ and Wang ${ }^{13}$ have applied the moment-averaged nematodynamic equations (hereafter called the Doi model) to predict spatially homogeneous nematic patterns that can be supported by an imposed, pure elongational velocity field $\mathbf{v}$ :

$$
\mathbf{v}=\nu\left(-\frac{x}{2},-\frac{y}{2}, z\right),
$$

given in rectangular coordinates $(x, y, z)$ with respect to the basis $\left(\mathbf{e}_{x}, \mathbf{e}_{y}, \mathbf{e}_{z}\right)$.

For $\nu>0$, the flow stretches along the $z$ axis which we call axial or unidirectional elongation; for $\nu<0$, the flow stretches radially in the entire plane orthogonal to the $z$ axis, which we call planar or bidirectional elongation.

The primary questions addressed in the seminal papers listed above consist of the following.

(1) How is the equilibrium isotropic-to-nematic phase transition at critical LCP concentration altered by the presence of a steady flow field?

(2) What types of steady-state nematic patterns are created by an imposed flow (here elongational)?

(3) How many distinct nematic patterns coexist at prescribed values of the dimensionless LCP density parameter $(N)$ and Peclet number (Pe, the ratio of elongational rate to LCP molecular relaxation rate)?

(4) What and where are the phase transition boundaries in $(N, \mathrm{Pe})$ ?

(5) What are the stability properties of all steady state patterns?

(6) What stable patterns co-exist at any fixed $(N, \mathrm{Pe})$ ?

Answers to such fundamental questions are clearly valuable in guiding or explaining experiments and manufacturing processes in which elongational flow is dominant. A striking example of success of the Doi averaged model is given by the flow reversal phenomenon in filament flows of LCPs shown experimentally ${ }^{26}$ and reproduced from numerical simulations of the Doi model. ${ }^{7}$ The above questions are independent of free boundary effects, and so are potentially easier to address.

Of particular interest here is the uniaxial versus biaxial nature both of the patterns that form, and of their modes of stability and instability. Recent experimental techniques ${ }^{27-29}$ allow full tensor resolution of $\mathbf{Q}$, so the analytical understanding of the uniaxial and biaxial behavior becomes necessary to properly interpret experimental data, and even to guide the data collection. Our interpretation of tensorial basis modes in terms of splay, bend, and twist is due to Shimada et al. ${ }^{19}$

\section{THREE-DIMENSIONAL FORMULATION}

\section{A. The moment-averaged Doi model}

We recall the moment-averaged equations governing orientation and flow of LCPs as developed in Bhave et al.: ${ }^{4}$

Conservation of linear momentum:

$$
\rho \frac{d}{d t} \mathbf{v}=\nabla \cdot \tau,
$$

where $\rho$ is the density of the polymeric liquid, $\mathbf{v}$ is the velocity, $\tau$ is the total stress tensor and $d / d t$ denotes the material derivative defined by $d / d t=\partial / \partial t+\mathbf{v} \cdot \nabla$. Note that external forces are ignored.

Incompressibility: 
$\nabla \cdot \mathbf{v}=0$.

Constitutive equation for stresses:

$$
\begin{aligned}
& \tau=-p \mathbf{I}+\hat{\tau}, \\
& \hat{\tau}=2 \eta \mathbf{D}+3 c k T[\mathbf{F}(\mathbf{Q})+2 \lambda(\nabla \mathbf{v}: \mathbf{Q})(\mathbf{Q}+\mathbf{I} / 3)], \\
& \mathbf{F}(\mathbf{Q})=(1-N / 3) \mathbf{Q}-N(\mathbf{Q} \cdot \mathbf{Q})+N(\mathbf{Q}: \mathbf{Q})(\mathbf{Q}+\mathbf{I} / 3),
\end{aligned}
$$

where $\mathbf{D}=\frac{1}{2}\left[\nabla \mathbf{v}+\nabla \mathbf{v}^{T}\right]$ is the rate-of-strain tensor, $\nabla \mathbf{v}$ has $i, j$ component $\partial \mathbf{v}_{i} / \partial x_{j}$ in Cartesian coordinates, $p$ is the pressure, $\eta$ is the solvent viscosity, $\lambda$ is a relaxation time associated with rotation of the dumbbell molecules, $c$ is the number of polymer molecules per unit volume, $N$ is the dimensionless polymer concentration which measures the strength of the intermolecular short-range Maier-Saupe potential, $\mathbf{Q}$ is the orientation tensor defined by

$$
\mathbf{Q}=\langle\mathbf{m} \otimes \mathbf{m}\rangle-\mathbf{I} / 3
$$

where $\mathbf{m}$ is a unit vector in the LCP molecular direction, the average $\langle(\boldsymbol{O})\rangle$ is with respect to a molecular probability density function consistent with rigid rod molecules, $k$ is the Boltzmann constant, and $T$ is absolute temperature. We note that the general orientation tensor $\mathbf{Q}$, defined by (5), is a symmetric, traceless, rank two tensor. This means that once coordinates are specified, $\mathbf{Q}$ is represented as a symmetric, traceless, $3 \times 3$ matrix, i.e., $\mathbf{Q}$ has five independent components in general. The orientation-dependent stress contribution in (4) corresponds to anisotropic viscoelastic behavior of LCP flows; the flow-orientation system is closed with the

Nematodynamic equation for $\mathbf{Q}$ :

$$
\begin{aligned}
\frac{d}{d t} \mathbf{Q}-\left(\nabla \mathbf{v} \cdot \mathbf{Q}+\mathbf{Q} \cdot \nabla \mathbf{v}^{T}\right)= & \frac{2}{3} \mathbf{D}-2(\nabla \mathbf{v}: \mathbf{Q})(\mathbf{Q}+\mathbf{I} / 3) \\
& -\frac{\sigma}{\lambda} \mathbf{F}(\mathbf{Q}),
\end{aligned}
$$

where $\mathbf{F}$ is defined in (4) and $\sigma$ is a dimensionless parameter describing the anisotropic drag that a molecule experiences as it moves relative to the solution $(0<\sigma \leqslant 1)$. The isotropic drag condition is $\sigma=1$, and the highly anisotropic drag limit is $\sigma=0$.

Remark: As in Refs. 4, 8, 9, and 13, we use the Doi closure approximation in the above averaged equations. Wang $^{13}$ has extensively studied the results for three types of closure rules, that of Doi, Hinch-Leal 1 (HL1), ${ }^{30}$ and Hinch-Leal 2 (HL2). ${ }^{30}$ His analysis is restricted to a uniaxial tensor $\mathbf{Q}$, i.e., a scalar order parameter assumption as in Ref. 9, the conclusion of Ref. 13 is that the Doi closure rule and HL2 closure rule are most consistent with full kinetic theory predictions for imposed elongational flows and a uniaxial $\mathbf{Q}$ tensor. The HL1 rule yields spurious uniaxial equilibria in elongation (Ref. 13) and so is not pursued here. Because of the rational and transcendental form of the HL2 closure rule, we restrict this study to the standard Doi closure which preserves the order of nonlinearity of moments. From this same model, Rey $^{8}$ captures biaxial equilibria and stability proper- ties not resolved in Refs. 9 and 13, while we capture full tensor stability properties (in particular, director instabilities) not resolved in Rey. ${ }^{8}$

\section{B. Representations for $\mathbf{Q}$}

The key to our analysis lies in understanding and manipulating the orientation tensor $\mathbf{Q}$. We pause here to recall two essential representations of $\mathbf{Q}$, how they correspond, and how special forms of $\mathbf{Q}$ (e.g., uniaxial, biaxial but with fixed directors) are explicitly characterized within each type of representation.

\section{Component representations for $Q$}

A component representation is a standard matrix representation with respect to a chosen coordinate system: $\mathbf{Q}$ $=\left(Q_{i j}\right)$ with $Q_{i j}=Q_{j i}$ and $\operatorname{tr}(\mathbf{Q})=0$, where $Q_{i j}$ are components with respect to a coordinate basis. Here we use rectangular coordinates for which $Q_{i j}=Q_{x_{i} x_{j}}$, with $\mathbf{Q}=\Sigma Q_{i j} \mathbf{e}_{i}$ $\otimes \mathbf{e}_{j}$. For these coordinates, the symmetric traceless matrices form a five-dimensional vector space with the basis:

$$
\begin{aligned}
& \mathbf{Q}^{(1)}=\left[\begin{array}{ccc}
1 & 0 & 0 \\
0 & 1 & 0 \\
0 & 0 & -2
\end{array}\right], \quad \mathbf{Q}^{(2)}=\left[\begin{array}{ccc}
-1 & 0 & 0 \\
0 & 1 & 0 \\
0 & 0 & 0
\end{array}\right], \\
& \mathbf{Q}^{(3)}=\left[\begin{array}{lll}
0 & 1 & 0 \\
1 & 0 & 0 \\
0 & 0 & 0
\end{array}\right], \\
& \mathbf{Q}^{(4)}=\left[\begin{array}{lll}
0 & 0 & 1 \\
0 & 0 & 0 \\
1 & 0 & 0
\end{array}\right], \quad \mathbf{Q}^{(5)}=\left[\begin{array}{lll}
0 & 0 & 0 \\
0 & 0 & 1 \\
0 & 1 & 0
\end{array}\right] .
\end{aligned}
$$

Then,

$$
\begin{aligned}
\mathbf{Q}= & \frac{Q_{11}+Q_{22}}{2} \mathbf{Q}^{(1)}+\frac{Q_{22}-Q_{11}}{2} \mathbf{Q}^{(2)}+Q_{12} \mathbf{Q}^{(3)} \\
& +Q_{13} \mathbf{Q}^{(4)}+Q_{23} \mathbf{Q}^{(5)} .
\end{aligned}
$$

In Refs. 6 and 19 this basis is instrumental in solving the linearized pure Doi theory, governed by (6) with $\nabla \mathbf{v}=0$, about isotropic and nematic equilibria. This basis has also been utilized by Sonnet et al. ${ }^{31}$ The basis $\left\{\mathbf{Q}^{(i)}\right\}_{i=1}^{5}$ is fixed, with the "orthogonality property" that $\operatorname{tr}\left(\mathbf{Q}^{(i)} \cdot \mathbf{Q}^{(j)}\right)$ is proportional to $\delta_{i j}$.

With respect to the bases (7) and (8), $\mathbf{Q}^{(1)}$ is a splay mode with respect to $\mathbf{e}_{z}, \mathbf{Q}^{(2)}$ and $\mathbf{Q}^{(3)}$ correspond to twist modes, $\mathbf{Q}^{(4)}$ and $\mathbf{Q}^{(5)}$ correspond to bend modes. ${ }^{19}$

\section{2. "Spectral" representations for $Q$}

Traditional "continuum" approaches to LCPs posit $\mathbf{Q}$ on the basis of its eigenvalues and eigenvectors, in contrast to its components. Since $\mathbf{Q}$ is symmetric, the eigenvectors of $\mathbf{Q}$ form an orthonormal basis of $R^{3}$. From the spectral theorem, ${ }^{32}$ if $d_{i}$ is the eigenvalue associated with eigenvector $\mathbf{n}_{i}$, then 


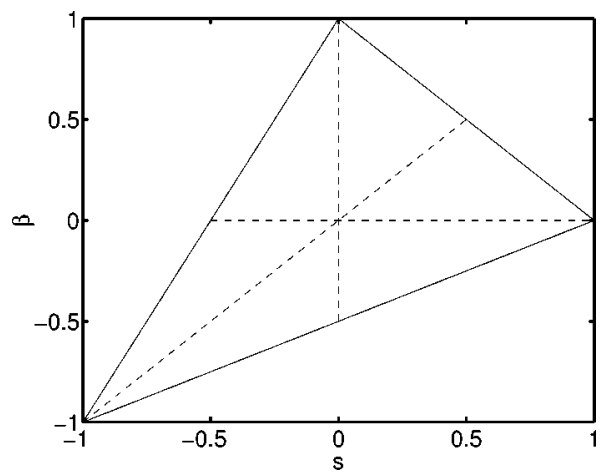

FIG. 1. The range of the pair of biaxial order parameters $s$ and $\beta$. The uniaxial limits are $\beta=0, s=0$, and $s=\beta$.

$$
\mathbf{Q}=\sum\left(d_{i}-\frac{1}{3}\right) \mathbf{n}_{i} \otimes \mathbf{n}_{i}, d_{i}=\left\langle\left(\mathbf{n}_{i} \cdot \mathbf{m}\right)^{2}\right\rangle, \sum d_{i}=1
$$

Since $\mathbf{Q}$ and $\langle\mathbf{m} \otimes \mathbf{m}\rangle$ share the same eigenvectors, $\left\{\mathbf{n}_{i}\right\}_{i=1}^{3}$ are the "directors" of the nematic LCP. The direction cosines $d_{i}$ characterize, in a nonlinear averaged sense, the degrees of orientation of the LCP molecules with respect to each director. These define two independent scalar order parameters,

$$
s=d_{3}-d_{1}, \beta=d_{2}-d_{1},
$$

which physically correspond to the degree of anisotropic order in the plane of $\left(\mathbf{n}_{3}, \mathbf{n}_{1}\right),\left(\mathbf{n}_{2}, \mathbf{n}_{1}\right)$, respectively. From (10), $\mathbf{Q}$ then admits a biaxial spectral representation:

$$
\mathbf{Q}=s\left(\mathbf{n}_{3} \otimes \mathbf{n}_{3}-\mathbf{I} / 3\right)+\beta\left(\mathbf{n}_{2} \otimes \mathbf{n}_{2}-\mathbf{I} / 3\right) .
$$

The range of $(s, \beta)$ is a closed triangular region in the $(s, \beta)$ plane; see Fig. 1. This representation is equivalent to the biaxial representation in Refs. 33 and 8.

\section{Uniaxial limits}

If all the eigenvalues of $\mathbf{Q}$ are distinct, the nematic LCP is fully biaxial. Uniaxial nematics are defined by the condition that two eigenvalues of $\mathbf{Q}$ are equal; the eigenvector (n) associated with the simple eigenvalue is distinguished, and called the uniaxial director. Since $\operatorname{tr}(\mathbf{Q})=0$ there is one independent eigenvalue, which defines a distinguished single order parameter.

The conditions for $\mathbf{Q}$ to be uniaxial take different forms. From the spectral representation (10), the uniaxial conditions are immediate:

$$
d_{1}=d_{2}, d_{1}=d_{3}
$$

or

$$
d_{2}=d_{3}
$$

in terms of the order parameters $(s, \beta)$ and Fig. 1:

$$
\begin{aligned}
& \beta=0, \text { with uniaxial order parameter } s_{u}=s \\
& \quad \text { and director } \mathbf{n}=\mathbf{n}_{3} ; \\
& s=0, \quad \text { with uniaxial order parameter } s_{u}=\beta \\
& \quad \text { and director } \mathbf{n}=\mathbf{n}_{2} ; \\
& s=\beta, \quad \text { with uniaxial order parameter } s_{u}=-s \\
& \quad \text { and director } \mathbf{n}=\mathbf{n}_{1} .
\end{aligned}
$$

In terms of the basis $\mathbf{Q}^{(i)}, \mathbf{Q}^{(1)}$ is the unique uniaxial tensor, corresponding in (12) to $\beta=0$, with director $\mathbf{n}_{3}=\mathbf{e}_{z}$, and uniaxial order parameter $s$, or equivalently, $\mathbf{Q}$ $=-(s / 3) \mathbf{Q}^{(1)}$. When $0<s \leqslant 1$, the liquid crystal exhibits "prolate" uniaxial symmetry; when $-1 / 2 \leqslant s<0$, there is "oblate" uniaxial symmetry; $s=-1 / 2$ corresponds to the LCP molecule aligned somewhere in the plane orthogonal to $\mathbf{n} ; s=1$ corresponds to parallel alignment of $\mathbf{n}$ and $\mathbf{m} ; s$ $=0$ corresponds to an isotropic state in which molecular orientation is equally probable in all directions.

\section{SPATIALLY HOMOGENEOUS PATTERNS IN IMPOSED ELONGATIONAL FLOWS}

Following Refs. 4, 8, 9, and 13, we impose a simple elongational flow, (1). The key observation of See et al. ${ }^{9}$ is that in rectangular coordinates, the velocity gradient is constant:

$$
\nabla \mathbf{v}=\nu \operatorname{diag}\left(-\frac{1}{2},-\frac{1}{2}, 1\right) .
$$

Therefore, the momentum equation (2), with $\hat{\tau}$ given by (4), is trivially satisfied if $\mathbf{Q}$ is independent of $\mathbf{x}$, i.e., for spatially homogeneous patterns. Moreover, since $\nabla \mathbf{v}$ is constant, the flow-driven Doi nematodynamic equation for spatially homogeneous $\mathbf{Q}$ in the presence of imposed elongational flow remains an autonomous tensor ordinary differential equation in the scaled time variable $\tilde{t}=(\sigma / \lambda) t,\left({ }^{\circ}\right)=(d / d \tilde{t})$ :

$$
\begin{aligned}
& \dot{\mathbf{Q}}=-\mathbf{F}(\mathbf{Q})+\mathbf{G}(\mathbf{Q} ; \widetilde{\mathbf{D}}), \\
& \mathbf{G}(\mathbf{Q} ; \widetilde{\mathbf{D}})=\widetilde{\mathbf{D}} \mathbf{Q}+\mathbf{Q} \widetilde{\mathbf{D}}+\frac{2}{3} \widetilde{\mathbf{D}}-2 \widetilde{\mathbf{D}}: \mathbf{Q}\left(\mathbf{Q}+\frac{\mathbf{I}}{3}\right),
\end{aligned}
$$

where

$$
\widetilde{\mathbf{D}}=\operatorname{Pe} \operatorname{diag}(-1 / 2,-1 / 2,1), \operatorname{Pe}=\frac{\nu \lambda}{\sigma} .
$$

Pe is the Peclet number, the critical flow/orientation dimensionless parameter, measuring the ratio of the elongational rate $\nu$ and the orientational relaxation time rate $\sigma / \lambda, \mathbf{F}(\mathbf{Q})$ is given in (4). Note that both $\mathbf{F}(\mathbf{Q})$ and $\mathbf{G}(\mathbf{Q} ; \widetilde{\mathbf{D}})$ are symmetric and traceless, therefore the fixed basis $\left\{\mathbf{Q}^{(i)}\right\}_{i=1}^{5},(7)$ and (8), remains valuable in identifying the special properties of (16); the coupled scalar form of (16) is given in Appendix A. It should be pointed out that in (16) $\mathbf{F}$ is based on the MaierSaupe intermolecular potential, but any quartic potential that 
reflects the $\mathrm{I}-\mathrm{N}$ transition yields to a similar analysis. Wang ${ }^{13}$ shows for uniaxial $\mathbf{Q}$ the different forms of $\mathbf{F}$ and $\mathbf{G}$ for different closure rules.

Observe that the flow-induced contribution, $\mathbf{G}(\mathbf{Q} ; \widetilde{\mathbf{D}})$, is a constant coefficient, quadratic polynomial perturbation of the cubic polynomial equation. Previous uniaxial ${ }^{9,13}$ and biaxial $^{4,8}$ order parameter analyses have exploited this fact by calculating perturbations of the pure nematic equilibria and their stability. All equilibria are continuations of the $\mathrm{Pe}=0$ case, since the number of complex equilibria of a system of cubic polynomial equations cannot increase under quadratic perturbation. follow.

We now list observations which guide the analysis to

(1) Without flow, all equilibria are uniaxial. ${ }^{6}$ (This fact applies only to quartic intermolecular potentials and to closure rules which preserve the degree of moments, e.g., Doi and HL1 closures.)

(2) With pure elongational flow, a simple continuation algorithm calculates all equilibria of (16). For $\mathrm{Pe}=0$, the critical concentrations are $N=8 / 3,3$, and all Pe-induced transitions in type (uniaxial versus biaxial), number, and stability of equilibria emerge from these two bifurcation points.

(3) For the elongation-driven model (16), the full symmetry by orthogonal $\mathbf{V}$ is broken, but a one-dimensional continuous symmetry is preserved for all orthogonal $\mathbf{V}$ that preserve the axis of flow symmetry $\mathbf{e}_{z}$,

$$
\mathbf{V}=\left(\begin{array}{lll}
\cos \zeta & \sin \zeta & 0 \\
-\sin \zeta & \cos \zeta & 0 \\
0 & 0 & 1
\end{array}\right)
$$

This observation is important in determining any degeneracy of equilibria of (16). In particular, since $\mathbf{Q}^{(1)}$ is a fixed point of the similarity transformation by $\mathbf{V}$,

$$
\mathbf{V} \mathbf{Q}^{(1)} \mathbf{V}^{T}=\mathbf{Q}^{(1)},
$$

all uniaxial equilibria of (16) are unique. All biaxial equilibria, however, will be seen to have one director parallel to $\mathbf{e}_{z}$, and thus all fully biaxial equilibria have a continuous symmetry corresponding to arbitrary orientation of the remaining two directors in the $x, y$ plane.

Contact with previous uniaxial ${ }^{9,13}$ and biaxial $^{8}$ order parameter equations for elongation-induced homogeneous patterns:

(1) The scalar order parameter equation of Refs. 9 and 13 follows by assuming $\mathbf{Q}$ proportional to $\mathbf{Q}^{(1)}$. The governing scalar order parameter equation is

$$
\dot{s}=\operatorname{Pe}\left[1+s-2 s^{2}\right]-U(s) .
$$

(2) The biaxial order parameter equations of Ref. 8 follow by assuming $\mathbf{Q}$ in the span of $\mathbf{Q}^{(1)}, \mathbf{Q}^{(2)}$, or equivalently, by positing $\mathbf{Q}$ of the form (12) with $\mathbf{n}_{3}=\mathbf{e}_{z}, \mathbf{n}_{2}=\mathbf{e}_{x}$ or $\mathbf{e}_{y}$. Note that solutions of (16) are invariant with respect to the similarity transform by $\mathbf{V}$, (18), thus each equilibrium $(s, \beta)$ corresponds to a family of equilibria, with $\mathbf{n}_{1}, \mathbf{n}_{2}$ in arbitrary position in the $x, y$ plane. This observation allows

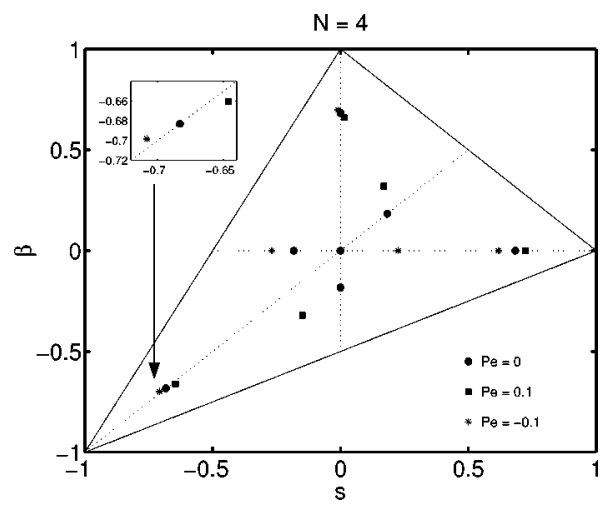

FIG. 2. Illustration of symmetry-broken genesis of biaxial equilibria for a fixed concentration $(N=4)$. Prior to the imposed flow $(\mathrm{Pe}=0)$, there are three uniaxial equilibria on each uniaxial subspace, $\beta=0, s=0, s=\beta$. With imposed axial $(\mathrm{Pe}=0.1)$ and planar $(\mathrm{Pe}=-0.1)$ elongation, the equilibria on the surviving uniaxial subspace $\beta=0$ survive as uniaxial steady states. However, the $s=0$ and $s=\beta$ uniaxial subspaces are destroyed, and their corresponding equilibria either deform into biaxial equilibrium patterns or are destroyed (become complex and therefore nonphysical).

us to fix the directors $\mathbf{n}_{1}, \mathbf{n}_{2}$ parallel to $\mathbf{e}_{x}, \mathbf{e}_{y}$, i.e., to fix $\zeta \equiv 0$. The governing biaxial order parameter equations are then

$$
s_{t}^{\sim}=\operatorname{Pe}\left[1-\beta+s+\beta s-2 s^{2}\right]-U(s)+\frac{2}{3} N s \beta(s-\beta-1),
$$

$$
\beta_{\tilde{t}}=\operatorname{Pe}\left[\beta^{2}-2 \beta s-\beta\right]-U(\beta)+\frac{2}{3} N s \beta(\beta-s-1) .
$$

Elongation-induced equilibrium patterns. The construction of all equilibria of (16) is straightforward. As noted by Rey, ${ }^{8}$ two of the uniaxial limits $s=0, s=\beta$, are no longer preserved by the nematic equation (16), but the fixed points that evolve from these uniaxial limits do survive, either as biaxial equilibria patterns or as nonreal, unphysical equilibria. Figure 2 illustrates the persistence of uniaxial equilibria whose director is parallel to $\mathbf{e}_{z}$ as well as the emergence of biaxial steady states for $\mathrm{Pe} \neq 0$ arising from the flowindependent uniaxial equilibria whose directors were not aligned with $\mathbf{e}_{z}$. This result is intuitively quite natural, and it is noteworthy that the Doi closure model captures the phenomenon precisely.

Stability of equilibria. From the above remarks, it follows that all changes in the number of equilibria occur within the order parameter equations (21). Their stability, however, requires analysis of the remaining three "director modes." This extension of the linearized stability of equilibria is the fundamental new contribution of this paper. The linearized stability for the order parameter system (21) is a straightforward $2 \times 2$ linear algebra calculation accessible by symbolic software; the linearized eigenvalues $\mu_{1}, \mu_{2}$ about any equilibrium $(s, \beta)=\left(s^{*}, \beta^{*}\right)$ are

$$
\begin{aligned}
\mu_{1}= & -\frac{3 \mathrm{Pe}}{2}(2 s-\beta)-\left[1-N / 3-4 N s \beta / 3+4 N \beta^{2} / 3\right. \\
& \left.+4 N s^{2} / 3-\sqrt{\Delta}\right],
\end{aligned}
$$




$$
\begin{aligned}
\mu_{2}= & -\frac{3 \mathrm{Pe}}{2}(2 s-\beta)-\left[1-N / 3-4 N s \beta / 3+4 N \beta^{2} / 3\right. \\
& \left.+4 N s^{2} / 3+\sqrt{\Delta}\right], \\
\Delta= & \frac{4}{9} N^{2}\left[s^{2}(s-1)^{2}+\beta^{2}(\beta-1)^{2}+3 s \beta(s+\beta+s \beta)\right. \\
& \left.-s \beta\left(2 s^{2}+2 \beta^{2}+1\right)\right]+\frac{2}{3} N \mathrm{Pe}\left[2 s(s-1)^{2}\right. \\
& \left.-\beta(\beta-1)^{2}+s \beta(4-3 s+3 \beta)\right]+\frac{1}{4} \operatorname{Pe}^{2}(2 s-\beta-2)^{2} .
\end{aligned}
$$

The corresponding linearized eigenfunctions consist of nontrivial linear combinations of $\mathbf{Q}^{(1)}, \mathbf{Q}^{(2)}$. Remarkably, the remaining three linearized eigenfunctions are precisely $\mathbf{Q}^{(3)}$, $\mathbf{Q}^{(4)}, \mathbf{Q}^{(5)}$, a fact which immediately yields explicit formulas for the linearized eigenvalues $\mu_{3}, \mu_{4}, \mu_{5}$ :

$\mu_{3}=-\operatorname{Pe}(1+2 s-\beta)-\left[\frac{U(s)}{s}+N s+\frac{N \beta}{3}(2 \beta-1-2 s)\right]$,

\begin{tabular}{|c|c|c|c|c|}
\hline Region & $\begin{array}{c}\text { Type of } \\
\text { steady states }\end{array}$ & 1-D subspace & 2-D subspace & Full 5-D space \\
\hline I: & $\mathbf{P}^{1}$ & 0 & 0 & 0 \\
\hline \multirow[t]{3}{*}{ II: } & $\mathbf{P}^{1}$ & 0 & 0 & 0 \\
\hline & $P^{2}$ & $1^{\mathrm{a}}$ & $1^{\mathrm{a}}$ & $1^{\mathrm{a}}$ \\
\hline & $\mathbf{P}^{3}$ & 0 & 0 & 0 \\
\hline \multirow[t]{7}{*}{ III: } & $\mathbf{P}^{1}$ & 0 & 0 & 0 \\
\hline & $P^{2}$ & $1^{\mathrm{a}}$ & $1^{\mathrm{a}}$ & $1^{\mathrm{a}}$ \\
\hline & $\mathbf{P}^{3}$ & 0 & 0 & 0 \\
\hline & $B^{1}$ & & 0 & $1^{\mathrm{d}}$ \\
\hline & $B^{2}$ & & $1^{\mathrm{f}}$ & $2^{\mathrm{f}, \mathrm{d}}$ \\
\hline & $B^{3}$ & & $1^{\mathrm{f}}$ & $2^{\mathrm{f}, \mathrm{e}}$ \\
\hline & $B^{4}$ & & 0 & $1^{\mathrm{e}}$ \\
\hline \multirow[t]{5}{*}{ IV: } & $\mathbf{P}^{1}$ & 0 & 0 & 0 \\
\hline & $B^{1}$ & & 0 & $1^{\mathrm{d}}$ \\
\hline & $B^{2}$ & & $1^{\mathrm{f}}$ & $2^{\mathrm{f}, \mathrm{d}}$ \\
\hline & $B^{3}$ & & $1^{\mathrm{f}}$ & $2^{\mathrm{f}, \mathrm{e}}$ \\
\hline & $B^{4}$ & & 0 & $1^{\mathrm{e}}$ \\
\hline \multirow[t]{7}{*}{ V: } & $O^{1}$ & $1^{\mathrm{a}}$ & $2^{a, b}$ & $5^{\mathrm{a}, \mathrm{b}, \mathrm{c}, \mathrm{d}, \mathrm{e}}$ \\
\hline & $O^{2}$ & 0 & $1^{\mathrm{b}}$ & $4^{\mathrm{b}, \mathrm{c}, \mathrm{d}, \mathrm{e}}$ \\
\hline & $\mathbf{P}^{1}$ & 0 & 0 & 0 \\
\hline & $B^{1}$ & & 0 & $1^{\mathrm{d}}$ \\
\hline & $B^{2}$ & & $1^{\mathrm{f}}$ & $2^{\mathrm{f}, \mathrm{d}}$ \\
\hline & $B^{3}$ & & $1^{\mathrm{f}}$ & $2^{\mathrm{f}, \mathrm{e}}$ \\
\hline & $B^{4}$ & & 0 & $1^{\mathrm{e}}$ \\
\hline
\end{tabular}

$\mu_{4}=-\frac{\mathrm{Pe}}{2}(-1+4 s-2 \beta)-\left[\frac{U(s)}{s}+\frac{N \beta}{3}(-1-2 s+2 \beta)\right]$,

TABLE I. Dimension of instability for all steady states in axial elongation flows.

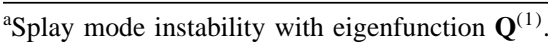

${ }^{\mathrm{b}}$ Twist mode instability with eigenfunction $\mathbf{Q}^{(2)}$

${ }^{c}$ Twist mode instability with eigenfunction $\mathbf{Q}^{(3)}$.

${ }^{\mathrm{d} B e n d}$ mode instability with eigenfunction $\mathbf{Q}^{(4)}$.

${ }^{e}$ Bend mode instability with eigenfunction $\mathbf{Q}^{(5)}$.

${ }^{\mathrm{f}}$ Mixed splay and twist mode instability with eigenfunction in the $\operatorname{span}\left\{\mathbf{Q}^{(1)}\right.$, $\left.\mathbf{Q}^{(2)}\right\}$.
$\mu_{5}=-\frac{\mathrm{Pe}}{2}(-1-2 \beta+4 s)-\left[\frac{U(s)}{s}+\frac{2 N \beta}{3}(1-s+\beta)\right]$.

Remark: Note that a restricted order parameter analysis of (21) fails to detect director instabilities. In Table I, the biaxial patterns $B^{1}, B^{4}$ in regions III-V each have an instability in one of the bend modes $\mathbf{Q}^{(4)}, \mathbf{Q}^{(5)}$.

All that remains then is to track the phase transitions (bifurcations) that we previously observed must emanate from $\mathrm{Pe}=0$ and the two bifurcation values $N=\frac{8}{3}$ and $N=3$. Of course, this continuation of the bifurcation branches is accomplished numerically.

Phase transitions emanating from $N=\frac{8}{3}, P e=0$ (Fig. $3)$. There are three distinct saddle-node $(\mathrm{SN})$ bifurcation points, $\left(s^{*}, \beta^{*}\right)=\left(\frac{1}{4}, 0\right)$, characterized by $\mu_{1}\left(s^{*}, \beta^{*}\right) \equiv 0$ and $\mu_{2}\left(s^{*}, \beta^{*}\right) \neq 0$; and $\left(s^{*}, \beta^{*}\right)=\left(0, \frac{1}{4}\right),\left(s^{*}, \beta^{*}\right)=\left(-\frac{1}{4},-\frac{1}{4}\right)$, characterized by $\mu_{1}\left(s^{*}, \beta^{*}\right) \neq 0, \mu_{2}\left(s^{*}, \beta^{*}\right) \equiv 0$. Their fate under imposed elongation is as follows.

(1) The SN $\left(\frac{1}{4}, 0\right)$ persists as a uniaxial SN bifurcation for $\mathrm{Pe} \neq 0$; see branch AFDJ, Fig. 3. Crossing AFDJ from left to right, for example by fixing the flow rate and increasing the concentration, the number of uniaxial equilibria jumps by two.

(2) The remaining two SN bifurcations, $\left(0, \frac{1}{4}\right)$ and $\left(-\frac{1}{4}\right.$, $\left.-\frac{1}{4}\right)$, persist as simultaneous biaxial SN bifurcations for $\mathrm{Pe} \neq 0$; see branch GBFE, Fig. 3. Crossing GBFE from left to right, the number of biaxial equilibria jumps by four.

Phase transitions emanating from $N=3, P e=0$ (Fig. 3). The isotropic equilibrium $s=\beta=0$ is doubly degenerate, i.e., both components of the polynomial vector field (21) vanish to second order, so that both $\mu_{1}$ and $\mu_{2}$ vanish absent of flow.

(1) The continuation of $\mu_{1}=0$ for Pe $>0$ is a uniaxial SN branch, $\mathrm{ABCH}$ of Fig. 3. Crossing $\mathrm{ABC}$ from left to right the

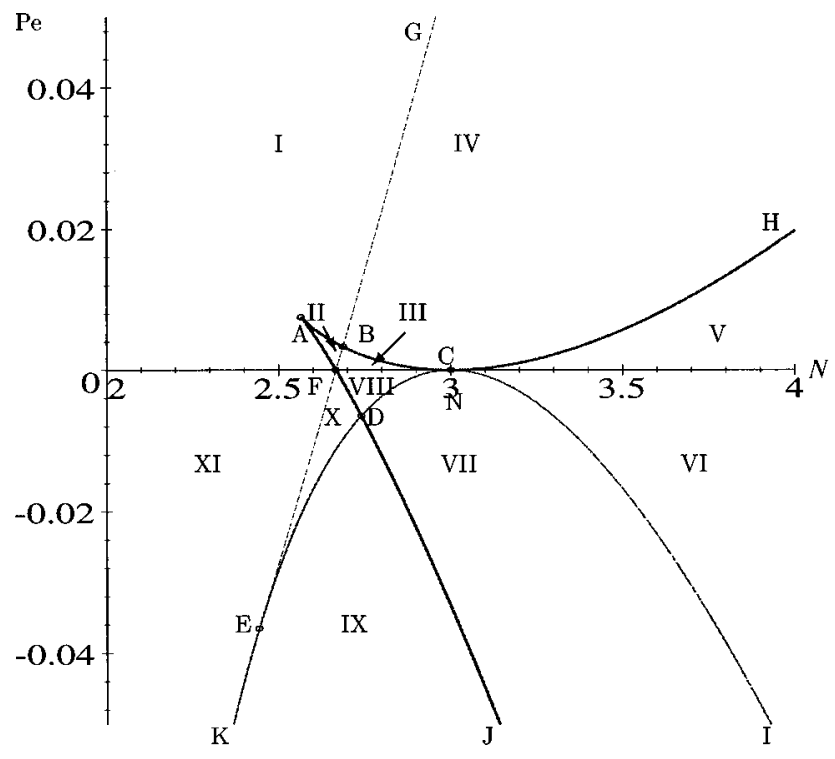

FIG. 3. Flow-driven phase diagram in the parameter space $(N, \mathrm{Pe})$.The phase transition curves correspond to loci in $(N, \mathrm{Pe})$ along which the linearized eigenvalues $\mu_{1}$ or $\mu_{2}$ vanish corresponding to degenerate equilibria. 
TABLE II. Dimension of instability for all steady states in planar elongation flows.

\begin{tabular}{|c|c|c|c|c|}
\hline Region & $\begin{array}{c}\text { Type of } \\
\text { steady states }\end{array}$ & 1-D subspace & 2-D subspace & Full 5-D space \\
\hline \multirow[t]{7}{*}{ VI: } & $O^{1}$ & 0 & $1^{\mathrm{b}}$ & $2^{\mathrm{b}, \mathrm{c}}$ \\
\hline & $P^{1}$ & 0 & 0 & $2^{\mathrm{d}, \mathrm{e}}$ \\
\hline & $P^{2}$ & $1^{\mathrm{a}}$ & $2^{a, b}$ & $5^{\mathrm{a}, \mathrm{b}, \mathrm{c}, \mathrm{d}, \mathrm{e}}$ \\
\hline & $\mathbf{B}^{1}$ & & 0 & 0 \\
\hline & $B^{2}$ & & $1^{\mathrm{f}}$ & $3^{\mathrm{f}, \mathrm{d}, \mathrm{e}}$ \\
\hline & $B^{3}$ & & $1^{\mathrm{f}}$ & $3^{\mathrm{f}, \mathrm{d}, \mathrm{e}}$ \\
\hline & $B^{4}$ & & 0 & 0 \\
\hline \multirow[t]{5}{*}{ VII: } & $O^{1}$ & 0 & $1^{\mathrm{b}}$ & $2^{b, c}$ \\
\hline & $P^{1}$ & 0 & 0 & $2^{\mathrm{d}, \mathrm{e}}$ \\
\hline & $P^{2}$ & $1^{\mathrm{a}}$ & $1^{\mathrm{a}}$ & $3^{\mathrm{a}, \mathrm{d}, \mathrm{e}}$ \\
\hline & $\mathrm{B}^{1}$ & & 0 & 0 \\
\hline & $\mathbf{B}^{4}$ & & 0 & 0 \\
\hline \multirow[t]{7}{*}{ VIII: } & $\mathbf{O}^{1}$ & 0 & 0 & 0 \\
\hline & $P^{1}$ & 0 & 0 & $2^{\mathrm{d}, \mathrm{e}}$ \\
\hline & $P^{2}$ & $1^{\mathrm{a}}$ & $1^{\mathrm{a}}$ & $3^{\mathrm{a}, \mathrm{d}, \mathrm{e}}$ \\
\hline & $\mathbf{B}^{1}$ & & 0 & 0 \\
\hline & $B^{1 u}$ & & $1^{\mathrm{f}}$ & $1^{\mathrm{f}}$ \\
\hline & $\mathbf{B}^{4}$ & & 0 & 0 \\
\hline & $B^{4 u}$ & & $1^{\mathrm{f}}$ & $1^{\mathrm{f}}$ \\
\hline \multirow[t]{3}{*}{ IX: } & $O^{1}$ & 0 & $1^{\mathrm{b}}$ & $2^{b, c}$ \\
\hline & $\mathbf{B}^{1}$ & & 0 & 0 \\
\hline & $B^{4}$ & & 0 & 0 \\
\hline \multirow[t]{5}{*}{$\mathrm{X}:$} & $\mathbf{O}^{1}$ & 0 & 0 & 0 \\
\hline & $\mathbf{B}^{1}$ & & 0 & 0 \\
\hline & $B^{1 u}$ & & $1^{\mathrm{f}}$ & $1^{\mathrm{f}}$ \\
\hline & $\mathbf{B}^{4}$ & & 0 & 0 \\
\hline & $B^{4 u}$ & & $1^{\mathrm{f}}$ & $1^{\mathrm{f}}$ \\
\hline XI: & $\mathbf{O}^{1}$ & 0 & 0 & 0 \\
\hline
\end{tabular}

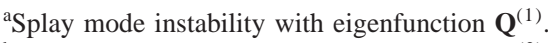

${ }^{\mathrm{b}}$ Twist mode instability with eigenfunction $\mathbf{Q}^{(2)}$.

${ }^{c}$ Twist mode instability with eigenfunction $\mathbf{Q}^{(3)}$.

${ }^{\mathrm{d}}$ Bend mode instability with eigenfunction $\mathbf{Q}^{(4)}$.

${ }^{\mathrm{e}}$ Bend mode instability with eigenfunction $\mathbf{Q}^{(5)}$.

Mixed splay and twist mode instability with eigenfunction in the $\operatorname{span}\left\{\mathbf{Q}^{(1)}\right.$ $\left.\mathbf{Q}^{(2)}\right\}$.

number of uniaxial equilibria jumps by two, then crossing $\mathrm{CH}$ from left to right the number of uniaxial steady states drops by two.

(2) The continuation of $\mu_{1}=0$ for $\mathrm{Pe}<0$ yields complex equilibria.

(3) The continuation of $\mu_{2}=0$ for $\mathrm{Pe}<0$ is a biaxial SN branch KEDCI of Fig. 3, corresponding to the broken uniaxial symmetry. Crossing KEDC from left to right, the number of biaxial equilibria jumps by two, then crossing CI from left to right the number of biaxial equilibria drops by two.

(4) The continuation of $\mu_{2}=0$ for Pe $>0$ yields complex equilibria.

The phase transition curves of Fig. 3, as described above, separate the $(N, \mathrm{Pe})$ half-plane into eleven distinct regions, labeled I-XI. For each region, Table I $(\mathrm{Pe}>0)$ and Table II $(\mathrm{Pe}<0)$ provide a list of all steady states, both uniaxial ( $P^{i}$ for each prolate, $O^{i}$ for each oblate, $i$ running over the number of such states) and biaxial $\left[B^{i}, i\right.$ running over each distinct biaxial order parameter pair $\left.\left(s^{*}, \beta^{*}\right)\right]$; the number of unstable tensorial eigenfunctions within each subspace that contains the given equilibrium; and finally, the superscripts convey the particular tensorial eigenfunctions for each instability. The stable equilibria, as deduced from the five-dimensional (5D) full stability analysis (the last column of Tables I and II), are listed in bold type.

Referring to Fig. 3 and Tables I and II, we summarize the major results.

(1) The symmetry (18), restricted to uniaxial equilibria (19), implies uniaxial steady states are discrete, or isolated, solutions of (16). For biaxial equilibria, however, similarity transform by $\mathbf{V}$, (18), maps any equilibrium with angle $\zeta$ $=0$ to arbitrary $\zeta$, maintaining the order parameters and moving the distinct biaxial directors $\mathbf{n}_{1}, \mathbf{n}_{2}$ to angle $\zeta$ with respect to $\mathbf{e}_{x}, \mathbf{e}_{y}$. Every biaxial equilibrium therefore corresponds to a continuous family with the corresponding zero linearized eigenvalue $\mu_{3}$. Strictly speaking, no biaxial patterns are linearly stable; some biaxial states have $\mu_{1}, \mu_{2}$, $\mu_{4}, \mu_{5}<0, \mu_{3}=0$, which is called center stable. Upon reflection, this result is also intuitively clear, since there is nothing in the flow or the LCP physics to select a distinguished direction in the $x-y$ plane. Again, the Doi closure model captures this phenomenon precisely.

(2) Regions I, IV, V, and XI have a unique, uniaxial, stable equilibrium. Note these are prolate phases for $\mathrm{Pe}>0$, and oblate for $\mathrm{Pe}<0$.

(3) Regions II and III, corresponding to axial elongation $(\mathrm{Pe}>0)$, have bistable prolate patterns.

(4) Regions VI, VII, and IX have center-stable biaxial patterns, with no uniaxial stable states. [We remark that biaxial patterns arise in pairs that are related through a similarity transformation $\mathbf{V}$, (18), with $\zeta=\pi / 2$. For example, in region VII, $B^{4}$ can be obtained from $B^{1}$ by rotating the two directors $\mathbf{n}_{1}, \mathbf{n}_{2}$ in the plane orthogonal to the flow direction by $\pi / 2$. This discrete symmetry implies that the continuous symmetry (18) applied to $B^{1}$ and $B^{4}$ yield identical continuous families.] Stable oblate uniaxial and center-stable biaxial patterns coexist in regions VIII and X.

(5) Biaxial order parameter instabilities of uniaxial states are revealed by a jump in the number of instabilities between the one-dimensional uniaxial and two-dimensional biaxial subspaces in Tables I and II. For example, in region V, the oblate phases $O^{1}$ and $O^{2}$ each acquire a biaxial order parameter instability in the twist mode $\mathbf{Q}^{(2)}$. A scalar uniaxial analysis misses these phenomena.

(6) Director instabilities are revealed by a jump in the number of unstable modes between the 2-D order parameter and full 5-D tensor spaces in Tables I and II.

(a) Director instabilities of uniaxial equilibria:

For $\mathrm{Pe}>0$, in region $V$ the two oblate phases $O^{1}, O^{2}$ are unstable to all director modes $\mathbf{Q}^{(i)}, i=3,4,5$.

(ii) For $\mathrm{Pe}<0$, in regions VI-VIII a pure order parameter analysis leads to the erroneous conclusion that one prolate equilibrium, labeled $P^{1}$, is stable. In fact $P^{1}$ is unstable to both bend modes $\mathbf{Q}^{(4)}, \mathbf{Q}^{(5)}$ in all three regions. Thus, no prolate patterns are stable in planar elongation.

(iii) The oblate phase $O^{1}$ in regions VI, VII, IX has a 
director instability in the twist mode $\mathbf{Q}^{(3)}$ as well as the order parameter instability in the twist mode $\mathbf{Q}^{(2)}$.

(iv) The prolate phase $P^{2}$ of region VI is unstable to all five orientation modes. The equilibria $P^{2}$ in regions VII, VIII have one splay mode instability, and two bend mode instabilities.

(b) Director instabilities of biaxial equilibria:

For $\mathrm{Pe}>0$, in regions III-V a pure order parameter analysis implies stable biaxial patterns $B^{1}, B^{4}-$ in fact, all of these states are unstable to one bend mode, $\mathbf{Q}^{(4)}, \mathbf{Q}^{(5)}$, respectively. The lower branches $B^{2}, B^{3}$ are unstable to one order parameter mixed mode, but also unstable to one bend mode. The upshot is that no biaxial patterns are stable to axial elongation.

(ii) For $\mathrm{Pe}<0$, stable biaxial patterns, labeled $B^{1}, B^{4}$, exist in regions VI-X. The "lower' biaxial branches $B^{2}, B^{3}$ in region VI are unstable to both bend (director) modes $\mathbf{Q}^{(4)}, \mathbf{Q}^{(5)}$.

\section{CONCLUSION}

From the Doi closure model with an imposed elongational flow, we pull together previous results and analyses (Refs. 19. 8, and 13) to give a complete phase diagram of homogeneous pattern selection: All elongation-induced equilibria, their linearized stability, all phase transitions, and the orientation modes and growth rates of every instability. Uniaxial equilibria are isolated, whereas all biaxial patterns arise as a continuous family with arbitrary directors in the plane orthogonal to the flow axis of symmetry.

From a stability perspective, we isolate order parameter and director modes of instability in the linearized analysis. Every fully biaxial equilibrium has one zero eigenvalue, corresponding to the rotational symmetry of directors in the $x-y$ plane transverse to the flow axis; the neutral orientation mode is always the twist mode $\mathbf{Q}^{(3)}$. Some patterns are stable to order parameter dynamics, and yet unstable to director modes, e.g., all biaxial patterns in axial elongation $(\mathrm{Pe}>0)$ suffer at least one bend mode instability. Thus, in axial elongation the only stable equilibrium patterns are uniaxial. In planar elongation, by contrast, above a moderate concentration and for arbitrary elongation rate, the only center-stable patterns are biaxial and there are no strictly stable patterns.

The phase diagram presented here is robust to the quadratic closure rule, in that any quadratic closure will simply modify the equilibria and stability. Since the Doi closure model can be identified with the Beris-Edwards continuum model, ${ }^{1}$ predictions on pattern selection are qualitatively similar. It is noteworthy that a Doi closure model with quartic intermolecular potential, or the analogous Beris-Edwards continuum theory model, yields intuitive predictions for homogeneous pattern selection in imposed uniaxial and planar elongation. We thereby anticipate these qualitative results can be used as a guide for processing windows in LCP concentration $(N)$ and flow rate $(\mathrm{Pe})$ in extensional and film manufacturing processes of LCPs.

\section{ACKNOWLEDGMENT AND DISCLAIMER}

Effort sponsored by the Air Force Office of Scientific Research, Air Force Materials Command, USAF, under Grant Nos. F49620-99-1-0003 and F49620-99-2-0008. The US Government is authorized to reproduce and distribute reprints for governmental purposes notwithstanding any copyright notation thereon. The views and conclusions contained herein are those of the authors and should not be interpreted as necessarily representing the official policies or endorsements, either expressed or implied, of the Air Force Office of Scientific Research or the US Government.

\section{APPENDIX: FULL 5D FLOW-DRIVEN EQUATIONS}

Let $\mathbf{Q}=\left(Q_{i j}\right)$. The equations [(16)] for the five independent components are

$$
\begin{aligned}
& \dot{Q}_{11}=\frac{1}{3}\left(-9 \mathrm{U}\left(Q_{11}\right)+6 Q_{11}-2 Q_{11} N-2 Q_{11}^{2} N\right. \\
& +Q_{12}^{2} N-6 Q_{12}^{2} Q_{11} N+Q_{13}^{2} N-6 Q_{13}^{2} Q_{11} N \\
& -2 Q_{22} Q_{11} N-6 Q_{22} Q_{11}^{2} N-2 Q_{22}^{2} N \\
& \left.-6 Q_{22}^{2} Q_{11} N-2 Q_{23}^{2} N-6 Q_{23}^{2} Q_{11} N\right) \\
& +\operatorname{Pe}\left(3 Q_{11}^{2}+3 Q_{11} Q_{22}+Q_{22}-\frac{1}{3}\right), \\
& \dot{Q}_{22}=\frac{1}{3}\left(-9 \mathrm{U}\left(Q_{22}\right)+6 Q_{22}-2 Q_{22} N-2 Q_{22}^{2} N+Q_{12}^{2} N\right. \\
& \text { - } 6 Q_{12}^{2} Q_{22} N+Q_{23}^{2} N-6 Q_{13}^{2} Q_{22} N \\
& -2 Q_{22} Q_{11} N-6 Q_{11} Q_{22}^{2} N-2 Q_{11}^{2} N \\
& \left.-6 Q_{11}^{2} Q_{22} N-2 Q_{13}^{2} N-6 Q_{23}^{2} Q_{22} N\right) \\
& +\operatorname{Pe}\left(3 Q_{22}^{2}+3 Q_{11} Q_{22}+Q_{11}-\frac{1}{3}\right), \\
& \dot{Q}_{13}=\frac{1}{3}\left(-6 Q_{13} Q_{11}^{2} N-6 Q_{22} Q_{13} Q_{11} N-6 Q_{22}^{2} Q_{13} N\right. \\
& -3 Q_{22} Q_{13} N-6 Q_{13} Q_{12}^{2} N+3 Q_{23} Q_{12} N \\
& -3 Q_{13}^{2} N-6 Q_{23}^{2} Q_{13} N-2 Q_{13} N-9 \mathrm{U}\left(Q_{13}\right) \\
& \left.+6 Q_{13}\right)+\operatorname{Pe} Q_{13}\left(3 Q_{11}+3 Q_{22}+\frac{1}{2}\right) \text {, } \\
& \dot{Q}_{12}=\frac{1}{3}\left(-6 Q_{12} Q_{11}^{2} N-6 Q_{22} Q_{12} Q_{11} N+3 Q_{12} Q_{11} N\right. \\
& -6 Q_{22}^{2} Q_{12} N+3 Q_{22} Q_{12} N-3 Q_{12}^{2} N \\
& -6 Q_{13}^{2} Q_{12} N-6 Q_{23}^{2} Q_{12} N-2 Q_{12} N \\
& \left.+3 Q_{23} Q_{13} N+6 Q_{12}-9 \mathrm{U}\left(Q_{12}\right)\right) \\
& +\operatorname{Pe} Q_{12}\left(3 Q_{11}+3 Q_{22}-1\right) \text {, } \\
& \dot{Q}_{23}=\frac{1}{3}\left(-6 Q_{23} Q_{11}^{2} N-6 Q_{23} Q_{22} Q_{11} N-3 Q_{23} Q_{11} N\right. \\
& \text { - } 6 Q_{23} Q_{22}^{2} N-6 Q_{23} Q_{12}^{2} N+3 Q_{13} Q_{12} N \\
& \text { - } 6 Q_{23} Q_{13}^{2} N-3 Q_{23}^{2} N-2 Q_{23} N-9 \mathrm{U}\left(Q_{23}\right) \\
& \left.+6 Q_{23}\right)+\operatorname{Pe} Q_{23}\left(3 Q_{11}+3 Q_{22}+\frac{1}{2}\right) .
\end{aligned}
$$


These equations can also be written in terms of the basis $\left\{\mathbf{Q}^{(i)}\right\}_{i=1}^{5}$, employing the expansion (9) and utilizing that $F$, (4), and $G,(16)$, consist of symmetric, traceless terms:

$$
\dot{\mathbf{Q}}=\mathbf{F}(\mathbf{Q})+\mathbf{G}(\mathbf{Q} ; \widetilde{\mathbf{D}})
$$

$$
\begin{aligned}
\mathbf{F}(\mathbf{Q})= & (1-N) \mathbf{Q}+N(\mathbf{Q}: \mathbf{Q}) \mathbf{Q}-\frac{N}{6}\left(2 Q_{12}^{2}-Q_{22}^{2}-Q_{23}^{2}-Q_{11}^{2}\right. \\
& \left.-Q_{13}^{2}-4 Q_{11} Q_{22}\right) \mathbf{Q}^{(1)}+\frac{1}{2}\left(Q_{11}^{2}+Q_{13}^{2}-Q_{22}^{2}-Q_{23}^{2}\right) \\
& \times \mathbf{Q}^{(2)}+\left(Q_{11} Q_{12}+Q_{12} Q_{22}+Q_{13} Q_{23}\right) \mathbf{Q}^{(3)} \\
& +\left(Q_{12} Q_{23}-Q_{13} Q_{22}\right) \mathbf{Q}^{(4)}+\left(Q_{12} Q_{13}-Q_{23} Q_{11}\right) \mathbf{Q}^{(5)},
\end{aligned}
$$

$$
\begin{aligned}
\mathbf{G}(\mathbf{Q} ; \widetilde{\mathbf{D}})= & -\frac{\operatorname{Pe}}{2}\left[-2 Q_{11} \mathbf{Q}^{(1)}+2\left(Q_{22}-Q_{11}\right) \mathbf{Q}^{(2)}\right. \\
& \left.+2 Q_{12} \mathbf{Q}^{(3)}-Q_{13} \mathbf{Q}^{(4)}-Q_{23} \mathbf{Q}^{(5)}\right] .
\end{aligned}
$$

${ }^{1}$ A. N. Beris and B. J. Edwards, Thermodynamics of Flowing Systems (Oxford University Press, Oxford, 1994).

${ }^{2}$ R. G. Larson, Constitutive Equations for Polymer Melts and Solutions (Butterworths, London, 1988).

${ }^{3}$ M. Doi and S. F. Edwards, The Theory of Polymer Dynamics (Oxford University Press, London, 1986).

${ }^{4}$ A. V. Bhave, R. K. Menon, R. C. Armstrong, and R. A. Brown, “A constitutive equation for liquid crystalline polymer solutions.' J. Rheol. 37, 413 (1993).

${ }^{5}$ M. G. Forest, Q. Wang, and S. E. Bechtel, "1-D models for thin filaments of liquid crystalline polymers: Coupling of orientation and flow in the stability of simple solutions," Physica D 99, 527 (1997).

${ }^{6} \mathrm{M}$. G. Forest, Q. Wang, and H. Zhou, "On phase transitions and pattern formation in nematic liquid crystal polymers," submitted to Phys. Rev. E (1999).

${ }^{7}$ N. Mori, Y. Hamaguchi, and K. Nakamura, "Numerical simulations of the spinning flow of liquid crystalline polymers," J. Rheol. 41, 1095 (1997).

${ }^{8} \mathrm{~A}$. Rey, "Bifurcational analysis of the isotropic-nematic phase transition of rigid rod polymers subjected to biaxial stretching flow," Macromol. Theory Simul. 4, 857 (1995).

${ }^{9}$ H. See, M. Doi, and R. G. Larson, "The effect of steady flow fields on the isotropic-nematic phase transition of rigid rod-like polymers," J. Chem. Phys. 92, 792 (1990).

${ }^{10}$ J. Feng, C. V. Chaubal, and L. G. Leal, "Closure approximations for the Doi theory: Which to use in simulating complex flows of liquid-crystalline polymers,' J. Rheol. 42, 1095 (1998).

${ }^{11}$ J. Feng and L. G. Leal, "Simulating complex flows of liquid-crystalline polymers using the Doi theory,"' J. Rheol. 41, 1317 (1997).

${ }^{12}$ J. Feng and L. G. Leal, "Pressure-driven channel flows of a model liquidcrystalline polymer,' Phys. Fluids 11, 2821 (1999).

${ }^{13} \mathrm{Q}$. Wang, "Comparative studies on closure approximations in flows of liquid crystal polymers: I. Elongational flows,' J. Non-Newtonian Fluid Mech. 72, 141 (1997)

${ }^{14} \mathrm{Q}$. Wang, "Biaxial steady states and their stability in shear flows of liquid crystal polymers," J. Rheol. 41, 943 (1997).

${ }^{15}$ A. Khokhlov and A. Semenov, "Influence of external field on the liquidcrystalline ordering in solutions of stiff-chain macromolecules," Macromolecules 15, 1272 (1982).

${ }^{16} \mathrm{~S}$. Wang and W. M. Gelbart, "Effect of elongational flow on the orientational order phase transitions and viscosity of hard rod fluids," J. Chem. Phys. 90, 597 (1989).

${ }^{17} \mathrm{~T}$. Hu and G. Ryskin, "Numerical simulation of rodlike polymers in a uniaxial extensional flow: The distribution-function version versus the order-parameter version of Doi's theory," J. Chem. Phys. 95, 6042 (1991).

${ }^{18} \mathrm{~T}$. Hu and G. Ryskin, "Numerical simulation of rodlike polymers in extensional and sink/source flows using the order-parameter version of Doi's theory," J. Chem. Phys. 96, 4705 (1992).

${ }^{19}$ T. Shimada, M. Doi, and K. Okano, "Concentration fluctuation of stiff polymers. III. Spinodal decomposition,'” J. Chem. Phys. 88, 7181 (1988).

${ }^{20}$ R. G. Larson and D. W. Mead, "Time and shear-rate scaling laws for liquid crystal polymers," J. Rheol. 33, 1253 (1989).

${ }^{21}$ R. G. Larson and M. Doi, "Mesoscopic domain theory for textured liquid crystalline polymers," J. Rheol. 35, 539 (1991).

${ }^{22} \mathrm{~T}$. Tsuji and A. Rey, "Orientation mode selection mechanisms for sheared nematic liquid crystalline materials," Phys. Rev. E 57, 5609 (1998).

${ }^{23}$ N. M. Kawaguchi and M. M. Denn, "A mesoscopic theory of liquid crystalline polymer,'’ J. Rheol. 43, 111 (1999).

${ }^{24} \mathrm{M}$. Doi, "Rheological properties of rodlike polymers in isotropic and liquid crystalline phases," Ferroelectrics 30, 247 (1980).

${ }^{25}$ M. Doi, "Molecular dynamics and rheological properties of concentrated solutions of rodlike polymers in isotropic and liquid crystalline phases," $\mathrm{J}$. Polym. Sci., Polym. Phys. Ed. 19, 229 (1981).

${ }^{26}$ N. Mori, Y. Hamaguchi, and K. Nakamura, "Measurement of velocity profile development in the spinning flows of liquid crystalline polymer solutions," J. Rheol. 41, 237 (1997).

${ }^{27}$ W. R. Burghardt, "Molecular orientation and rheology in sheared lyotropic liquid crystalline polymers," Macromol. Chem. Phys. 199, 471 (1998).

${ }^{28}$ S. G. Kalogrianitis and J. W. van Egmond, "Full tensor optical rheometry of polymer fluids,' J. Rheol. 41, 343 (1997).

${ }^{29}$ N. J. Wagner and L. M. Walker, "Determination of the texture viscosity and elasticity of a nematic PBLG/ $d=$ DMF solution through magnetic field alignment," Macromolecules 27, 5979 (1994).

${ }^{30}$ E. J. Hinch and L. G. Leal, "Constitutive equations in suspension mechanics. 2. Approximate forms for a suspension of rigid particles affected by Brownian rotations," J. Fluid Mech. 76, 187 (1976).

${ }^{31}$ A. Sonnet, A. Kilian, and S. Hess, "Alignment tensor versus director: Description of defects in nematic liquid crystals," Phys. Rev. E 52, 718 (1995).

${ }^{32}$ G. Strang, Linear Algebra and its Applications (Harcourt Brace, Jovanovitch, San Diego, 1986).

${ }^{33}$ G. Vertogen and W. H. deJeu, Thermotropic Liquid Crystals, Fundamentals, Springer Series in Chemical Physics, Vol. 45 (Springer, Berlin, 1988). 